\title{
Comparison of Adults with Attention-Deficit Hyperactivity Disorder Depending on the Age of Being Diagnosed in Childhood and Adulthood: Based on Retrospective Review in One University Hospital
}

\author{
Seong Woo Cho', Yeon Jung Lee ${ }^{2}$, Seong Ae Lee ${ }^{3}$, Minha Hong ${ }^{4}$, \\ Sang Min Lee ${ }^{5}$, Jin Cheol Park ${ }^{6}$, and Geon Ho Bahn \\ ${ }^{1}$ Department of Medicine, Graduate School, Kyung Hee University, Seoul, Korea \\ ${ }^{2}$ Department of Psychiatry, Seoul Hospital, Soonchunhyang University College of Medicine, Seoul, Korea \\ ${ }^{3}$ Department of Psychiatry, Kyung Hee University Hospital, Seoul, Korea \\ ${ }^{4}$ Department of Psychiatry, Seonam University College of Medicine, Myongji Hospital, Goyang, Korea \\ ${ }^{5}$ Department of Psychiatry, Konyang Univerisity College of Medicine, Konyang University Hospital, Daejeon, Korea \\ ${ }^{6}$ Department of Psychiatry, Silverheals Hospital, Namyangju, Korea \\ ${ }^{7}$ Department of Psychiatry, Kyung Hee University School of Medicine, Seoul, Korea
}

\begin{abstract}
Objectives: The study aimed to identify the characteristics of attention-deficit hyperactivity disorder (ADHD) that was not diagnosed in childhood or adolescence, but only in adulthood.

Methods: The characteristics of patients diagnosed with ADHD in adulthood were compared with those of patients diagnosed in childhood were assessed via a retrospective review of the medical records at one university hospital from 2005 to 2013 . If the age at which they were confirmed as having ADHD was less than 19 years old, they were grouped as childhood-diagnosed group (CD); if they were 19 years old or more, they were grouped as adulthood-diagnosed group (AD).

Results: The CD and AD included 50 (46.3\%) and 58 (53.7\%) patients, respectively. Inattention was the most common symptom in both groups. Behavioral and emotional problems were the second most frequent symptoms in the CD and AD, respectively. The intelligent quotient was significantly higher in the $\mathrm{AD}$ than in the $\mathrm{CD}$. The most common comorbidity was depression in the $\mathrm{CD}$ and personality disorder in the $\mathrm{AD}$. The most common reason for visiting the hospital was referral by acquaintances in the $\mathrm{CD}$ and media coverage in the $\mathrm{AD}$.

Conclusion: Clinicians should put ADHD on the index of suspicion when they examine adults with various psychiatric symptoms, because the diagnosis of ADHD might have been missed in childhood and the symptoms of ADHD might have changed as they grew up.
\end{abstract}

Key Words: Attention-deficit hyperactivity disorder; Diagnosis; Adult; Media; Childhood; Referral.

Received: May 6, 2017 / Revision: June 8, 2017 / Accepted: June 12, 2017

Address for correspondence: Geon Ho Bahn, Department of Psychiatry, Kyung Hee University School of Medicine, 23 Kyungheedae-ro, Dongdaemun-gu, Seoul 02447, Korea

Tel: +82-2-958-8556, Fax: +82-2-957-1997, E-mail: mompeian@khu.ac.kr

\section{INTRODUCTION}

While attention-deficit hyperactivity disorder (ADHD) was referred to as a disorder first diagnosed in infancy, childhood, and adolescence by the Diagnostic and Statistical Manual of Mental Disorders (DSM)-IV, ${ }^{1)}$ it is classified as one of neurodevelopmental disorders regardless of age in the DSM-5. ${ }^{2)}$ Interest in adults with ADHD is rising due to the increasing number of chronic ADHD cases and new hypotheses of lat-

This is an Open Access article distributed under the terms of the Creative Commons Attribution Non-Commercial License (http://creativecommons.org/licenses/by-nc/4.0) which permits unrestricted non-commercial use, distribution, and reproduction in any medium, provided the original work is properly cited. er-onset ADHD in adulthood or adolescence. ${ }^{3)}$ ADHD symptoms are noticed from early childhood and about half of patients have suffered from symptoms until adulthood. ${ }^{3)}$

The diagnosis of ADHD in patients who first visit the hospital as adult remains challenging. One of the reasons that diagnosis is difficult is that the clinical characteristics of ADHD might differ according to age. ${ }^{4)}$ Thus, if individuals have not been diagnosed with ADHD in childhood, it becomes very difficult to diagnose them in adulthood. Clinicians can resist an ADHD diagnosis, or preferentially diagnose affective disorders, or generalized anxiety disorder instead. That is, ADHD patients who are not diagnosed in childhood may experience 
secondary functional and psychological disorders ${ }^{4)}$ during adulthood. For patients with subthreshold symptoms who do not satisfy all the ADHD diagnostic criteria, the partial symptoms of ADHD can have a serious effect on their adult life. ${ }^{5)}$ Although many studies have investigated patients diagnosed with ADHD in childhood, little research is available regarding ADHD patients diagnosed in adulthood. ${ }^{3)}$

Clinicians who are familiar with the clinical characteristics of ADHD that appear in childhood as well as adulthood are best equipped to diagnose $\mathrm{ADHD}$ in patients who visit the hospital for the first time in adulthood and best positioned to establish optimal treatment plans. In this study, we examined the characteristics of adult patients who were diagnosed with ADHD first in adulthood, and compared them with adult patients who were diagnosed with ADHD in childhood and had been receiving treatment until they reached adulthood.

\section{METHODS}

\section{Subjects}

We selected the cases from the hospital records: patients 1) with ADHD who met the DSM-IV ${ }^{1)}$ criteria, 2) aged 19 and older, and 3) conducted outpatient visits more than three times at the department of psychiatry of a university hospital between January 2005 and December 2013. Diagnosis was done by one board-certified child psychiatrist. During outpatient visits, patients were assessed with Kiddie-Schedule for Affective Disorders and Schizophrenia-Present and LifetimeKorean version (K-SADS-PL-K), ${ }^{6}$ comprehensive attention test, ${ }^{7)}$ intelligence tests, ${ }^{8,9)}$ Temperament and Character Inventory (TCI), ${ }^{10)}$ and adult ADHD Self-Report Scale (ASRS) ${ }^{11)}$ beyond the DSM-IV criteria. 153 subjects were selected. Key exclusion criteria included mental retardation, pervasive developmental disorders, epilepsy or traumatic brain injury, a history of schizophrenia, mood disorder, or other severe mental disorders as the main diagnosis instead of ADHD. After thorough review, we included 108 patients, 84 males (77.8\%) and 20 females. Ages ranged from 19 to 63 years old.

To compare the characteristics of patients according to the time of diagnosis, we divided the subjects into two groups according to the age at which they were first diagnosed with ADHD. Those, who were diagnosed for the first time under 19 years of age and have been continuing treatment beyond 19 years old were classified into the childhood-diagnosed group (CD), and those, who were first diagnosed over 19 years of age and began treatment at that time were classified into the adulthood-diagnosed group (AD). The cutoff age of 19 was based on the standard for adulthood according to civil law. ${ }^{12)}$ This study was approved by Kyung Hee University Hospital
Institutional Review Board (IRB; KMC IRB 1341-08).

\section{Methods}

We verified the demographic and clinical characteristics via retrospective review of hospital records. The demographic variables including age, sex, treatment period and route of referral were assessed based on the first visit. To understand the clinical characteristics, we analyzed chief complaints, subtypes, comorbidity, intelligence quotient (IQ), and TCI scores. For chief complaints, two main complaints per person at the first visit were collected and classified as inattention, hyperactivity, impulsivity, emotional problems, behavioral problems, academic problems, interpersonal problems, addiction problems, evaluation of the individual's mental status and others. Others included complaints such as physical discomfort (e.g., headache, abdominal discomfort, and other somatic complaints), nail biting, and sleep problems. Subtypes of ADHD were classified as in DSM-IV.) Beyond ADHD, the second and the third diagnostic impressions were counted as comorbid diagnosis as the first visit and even during the follow-up period.

For age 6-16, the Korean-Wechsler Intelligence Scale for Children-III (K-WISC-III) ${ }^{8)}$ was administered. And for adolescents and adults aged 16-64, the Korean-Wechsler Adult Intelligence Scale-Revised (K-WAIS-R) ${ }^{9)}$ was administered. The $\mathrm{TCI},{ }^{10)}$ four temperament subscales, namely novelty seeking (NS), harm avoidance (HA), reward dependence (RD), and persistence $(\mathrm{P})$, and three character subscales, namely self directedness (SD), cooperativeness (C), and self transcendence (ST), was also administered. Higher subscale scores indicate a higher tendency toward the corresponding temperament or character trait. In this study, domestically standardized $\mathrm{TC}^{10)}$ for adults, adolescents, and children were used. IQ and TCI were analyzed only the results from the initial assessment.

\section{Statistical analysis}

Demographic and clinical characteristics were compared between $\mathrm{CD}$ and $\mathrm{AD}$. Independent two-sample t-tests were used for continuous variables, and chi-square tests were used for categorical variables. Statistical analyses were performed using SPSS 18.0 (SPSS Inc., Chicago, IL, USA), and the significance level was set at $\mathrm{p}<0.05$.

\section{RESULTS}

Mean age at the first visit was $13.8 \pm 3.2$ years for $C D(n=50)$ and 27.6 \pm 8.9 years for $\mathrm{AD}(\mathrm{n}=58)$ (Table 1). Male was predominant sex, $78.0 \%$ in $\mathrm{CD}$ and $77.6 \%$ in $\mathrm{AD}$. The average treatment period for $\mathrm{CD}$ was $70.4 \pm 43.8$ months, which was 
significantly longer than the period of $12.3 \pm 20.6$ months for $\mathrm{AD}(\mathrm{p}<0.01)$. In $\mathrm{CD}$, the hospital visit was most commonly the result of referral by acquaintances $(n=30,60.0 \%)$, followed by clinician's referral $(n=15,30.0 \%)$, and due to media coverage (internet, newspaper, TV, books, etc.) $(n=5,10.0 \%)$ (Table 1). In $A D$, media coverage ( $n=25,43.1 \%)$ was the most common reason for the first visit, followed by clinician's referral $(n=22,37.9 \%)$, and referral by acquaintances $(n=11,19.0 \%)$, revealing a significant difference between the two groups in route of referral $(\mathrm{p}<0.01)$.

Regarding the age distribution at the first visit, there were

Table 1. Demographic characteristics of subjects (\%)

\begin{tabular}{llc}
\hline \multicolumn{1}{c}{ Factors } & $\mathrm{CD}(\mathrm{n}=50)$ & $\mathrm{AD}(\mathrm{n}=58)$ \\
\hline $\begin{array}{l}\text { Mean age at first visit (years)* } \\
\text { Sex }\end{array}$ & $13.8 \pm 3.2$ & $27.6 \pm 8.9$ \\
$\quad$ Male & $39(78.0)$ & $45(77.6)$ \\
$\quad$ Female & $11(22.0)$ & $13(22.4)$ \\
Period of treatment (months)* & $70.4 \pm 43.8$ & $12.3 \pm 20.6$ \\
Route of referral* & & \\
$\quad$ Acquaintances & $30(60.0)$ & $11(19.0)$ \\
By clinicians & $15(30.0)$ & $22(37.9)$ \\
$\quad$ Media coverage & $5(10.0)$ & $25(43.1)$ \\
\hline *p<0.01. AD: adulthood-diagnosed group, CD: childhood-di- \\
agnosed group
\end{tabular}

15 elementary school students (aged from 7 to 12 years old), 20 middle school students (aged from 13 to 15 years old) and 15 high school students (aged from 16 to 18 years old) in the $\mathrm{CD}$ (Fig. 1). For the $\mathrm{AD}$, the most frequent age of the first visit was 19 years old. The oldest was a female who was first diagnosed in her early sixties.

Inattention was the most frequent chief complaint in both groups (Table 2). The second most frequent symptom was behavioral problems $(\mathrm{n}=14,28.0 \%)$ in $\mathrm{CD}$, and emotional problems $(n=23,39.7 \%)$ in AD. An initial visit for evaluation of individual's mental status was also reported $(n=13,22.4 \%)$ in $\mathrm{AD}$, especially males only.

In $\mathrm{CD}$, the subtypes in order of frequency were inattentive $(n=22,44.0 \%)$, not otherwise specified $(n=14,28.0 \%)$, combined ( $n=13,26.0 \%)$, and hyperactive/impulsive $(n=1,2.0 \%)$ (Table 2). In $\mathrm{AD}$, these were combined ( $\mathrm{n}=20,34.5 \%)$, inattentive ( $n=19,32.7 \%)$, not otherwise specified ( $n=15,25.9 \%)$, and hyperactive/impulsive $(n=4,6.9 \%)$ subtypes. There was no significant difference in subtype between groups.

The most common comorbidity was depressive disorder in $\mathrm{CD}(\mathrm{n}=21,42.0 \%)$, and personality disorder in $\mathrm{AD}(\mathrm{n}=16,27.6 \%)$ (Table 2). The number of comorbidities for CD was significantly higher than for $\mathrm{AD}$ (1.42 vs. 0.93, p<0.01).

Mean full scale IQ, verbal IQ, and performance IQ scores

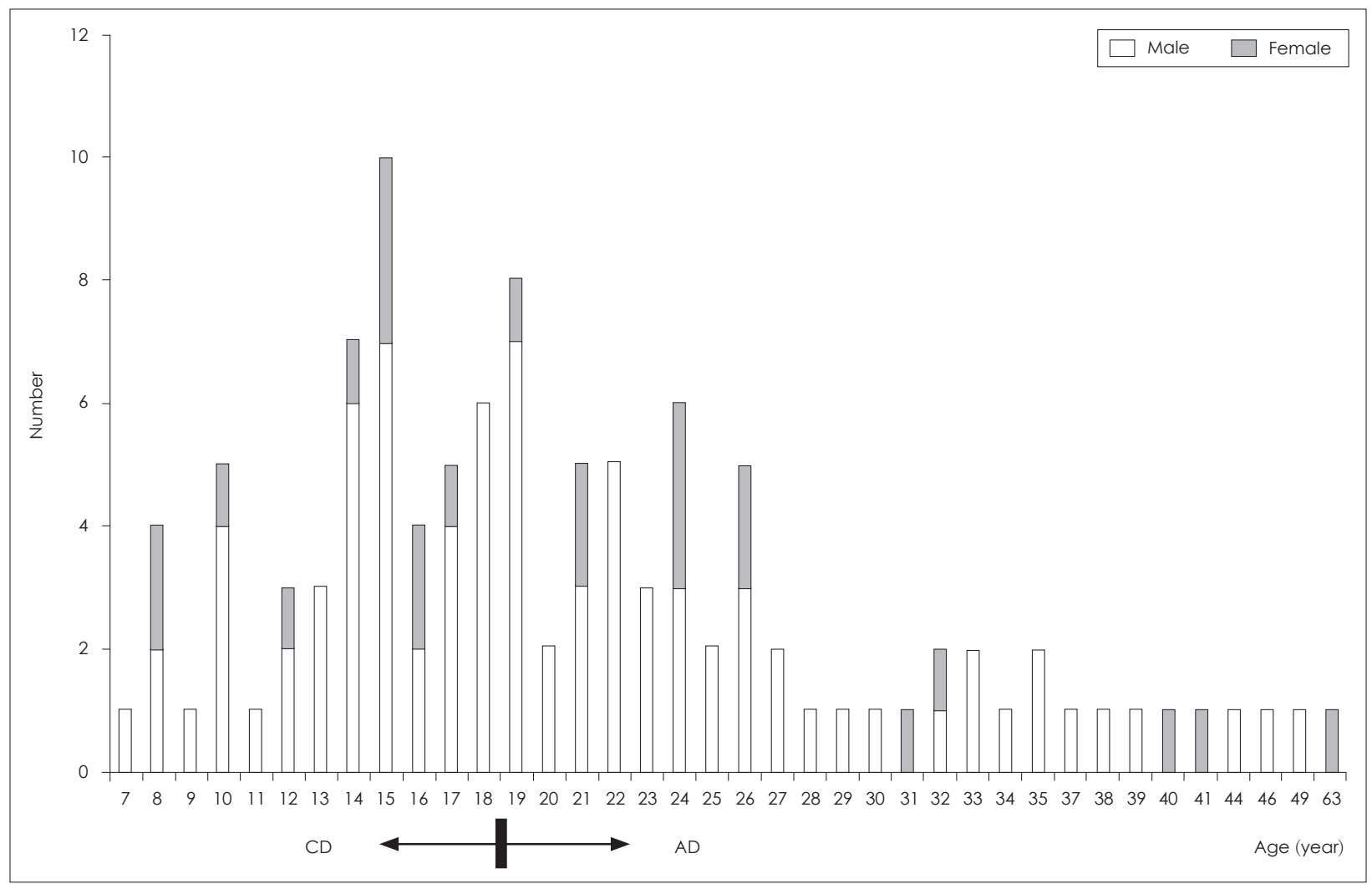

Fig. 1. Age distribution of adults diagnosed as ADHD. AD: adulthood-diagnosed group, ADHD: attention-deficit hyperactivity disorder, CD: childhood-diagnosed group. 
were 91.5, 92.7, and 92.1 for CD, and 106.1, 105.1, and 107.7 for $A D$, respectively (Table 3). IQs were significantly higher for $\mathrm{AD}$ than in $\mathrm{CD}(\mathrm{p}<0.01)$ (Table 3$)$.

Compared to $\mathrm{CD}, \mathrm{AD}$ was associated with significantly higher scores for the NS, HA subscales and significantly lower scores for the SD and C subscales (Table 3). There were no significant differences in RD, P, and ST between the two groups.

\section{DISCUSSION}

This study showed different demographic and clinical characteristics of ADHD depending on the age of being diagnosed as ADHD first time, even the patients were diagnosed with the same disorder and suffered untill adulthood.

According to the Health Insurance Review and Assessment Service in Korea which has data for all individuals aged 6-18, the mean age at which patients are first diagnosed with ADHD and start pharmacological treatment is $10.33 \pm 3.32$ years old, ${ }^{13)}$ which is approximately 3 years younger than the mean age in childhood diagnosed patients from this study. The reason for this discrepancy might be explained as a lower age at initial treatment is associated with a higher treatment discon- tinuation rate. ${ }^{14)}$ Also, subjects who started treatment at an early age may have been excluded because of treatment discontinuation and not included in this study.

Table 3. Comparison of $I Q$, temperament and character (mean \pm standard deviation)

\begin{tabular}{lccc}
\hline & $C D(n=49)$ & $A D(n=42)$ & $\dagger$ \\
\hline IQ & & & \\
FIQ & $91.5 \pm 20.4$ & $106.1 \pm 15.7$ & $-3.80^{\dagger}$ \\
VIQ & $92.7 \pm 21.0$ & $105.1 \pm 16.4$ & $-3.08^{\dagger}$ \\
PIQ & $92.1 \pm 19.0$ & $107.7 \pm 14.9$ & $-4.31^{\dagger}$ \\
TCl & & & \\
NS & $54.1 \pm 11.3$ & $63.4 \pm 15.0$ & $-2.98^{\dagger}$ \\
HA & $54.0 \pm 14.0$ & $61.5 \pm 14.6$ & $-2.22^{*}$ \\
RD & $44.9 \pm 12.0$ & $44.4 \pm 14.4$ & 0.15 \\
P & $39.0 \pm 11.2$ & $36.4 \pm 10.8$ & 1.03 \\
SD & $43.2 \pm 14.2$ & $33.5 \pm 12.6$ & $3.07^{\dagger}$ \\
C & $44.5 \pm 13.1$ & $34.7 \pm 15.4$ & $2.91^{\dagger}$ \\
ST & $46.5 \pm 13.2$ & $45.5 \pm 11.3$ & 0.36 \\
\hline
\end{tabular}

${ }^{*} p<0.05,{ }^{\dagger} p<0.01$. AD: adulthood-diagnosed group, C: cooperativeness, CD: childhood-diagnosed group, FIQ: full scale IQ, HA: harm avoidance, IQ: intelligence quotient, NS: novelty seeking, P: persistence, PIQ: performance IQ, RD: reward dependence, SD: self directedness, ST: self transcendence, TCl: Temperament Character Inventory, VIQ: verbal IQ

Table 2. Comparison of clinical characteristics

\begin{tabular}{|c|c|c|c|c|}
\hline Factors & $C D(n=50)$ & $\mathrm{n}(\%)$ & $A D(n=58)$ & $\mathrm{n}(\%)$ \\
\hline Chief complaints & Inattention & $16(32.0)$ & Inattention & $30(51.7)$ \\
\hline \multirow[t]{9}{*}{ at the first visit } & Behavioral problems & $14(28.0)$ & Emotional problems & $23(39.7)$ \\
\hline & Hyperactivity & $12(24.0)$ & For evaluation & $13(22.4)$ \\
\hline & Academic problems & $10(20.0)$ & Impulsivity & $8(13.8)$ \\
\hline & Interpersonal problem & $7(14.0)$ & Interpersonal problems & $7(12.1)$ \\
\hline & Emotional problems & $7(14.0)$ & Behavioral problems & $7(12.1)$ \\
\hline & Impulsivity & $5(10.0)$ & Hyperactivity & $6(10.3)$ \\
\hline & Addiction problems & $4(8.0)$ & Addiction problems & $5(8.6)$ \\
\hline & For evaluation & $4(8.0)$ & Academic problems & $3(5.2)$ \\
\hline & Others & $14(28.0)$ & Others & $5(8.6)$ \\
\hline \multirow[t]{4}{*}{ Subtype } & Inattentive & $22(42.0)$ & Combined & $20(34.5)$ \\
\hline & Not otherwise specified & $14(28.0)$ & Inattentive & $19(32.7)$ \\
\hline & Combined & $13(26.0)$ & Not otherwise specified & $15(25.9)$ \\
\hline & Hyperactive/impulsive & $1(2.0)$ & Hyperactive/impulsive & $4(6.9)$ \\
\hline \multirow[t]{10}{*}{ Comorbidity } & Depressive disorder & $21(42.0)$ & Personality disorder & $16(27.6)$ \\
\hline & Tic disorder & $9(18.0)$ & Depressive disorder & $10(17.2)$ \\
\hline & Personality disorder (trait) & $8(16.0)$ & Substance use disorder & $7(12.1)$ \\
\hline & Others & $8(16.0)$ & Anxiety disorder & $5(8.6)$ \\
\hline & Adjustment disorder & $7(14.0)$ & Adjustment disorder & $5(8.6)$ \\
\hline & DBD & $6(12.0)$ & Others & $4(6.9)$ \\
\hline & MR & $5(10.0)$ & Tic disorder & $3(5.1)$ \\
\hline & PDD & $4(8.0)$ & PDD & $3(5.1)$ \\
\hline & Substance use disorder & $2(4.0)$ & MR & $1(1.7)$ \\
\hline & Anxiety disorder & $1(2.0)$ & DBD & $0(0)$ \\
\hline
\end{tabular}

AD: adulthood-diagnosed group, CD: childhood-diagnosed group, DBD: oppositional defiant disorder, conduct disorder, MR: mental retardation, PDD: pervasive developmental disorder 
The gender proportion in this study was inconsistent with recent studies of the ADHD gender ratio, especially in adults, which proposed that the incidence of ADHD in females increases as the patients approach adulthood. ${ }^{15)}$ A strong hypothesis for the inconsistency of gender in adults from this study was culturally unique reason such as 'for the evaluation of the individual's mental state'. Although young boys or adolescents show the impulsivity and distraction, signs of ADHD, parents avoid visiting the psychiatrists because they are afraid of the stigma. However, as South Korea has the policy of mandatory enlistment for all men above 18 years old, young men with the probability of ADHD without previous diagnosis and their parents want to examine the mental state whether young men can adjust the military service. ${ }^{16)}$

Considering that the average age of $\mathrm{CD}$ at the time of this study was over 19 years old and that the monitoring period was $70.4 \pm 43.8$ months, the subjects in $\mathrm{CD}$ might have more severe symptoms and less responsive to treatment than typical children. It means that comorbid conditions may lead to the parents' compliance to pharmacotherapy. ${ }^{17)}$ Patients with comorbid conditions were more likely to adhere compared with reference variables such as age, sex, or parents education level. A monitoring study of a large-scale population group revealed that when ADHD was comorbid with behavioral disorders in adolescence, patients tended to be more susceptible to mood disorders compared to the general population or those with ADHD only. ${ }^{18)}$ The frequency of comorbidity was higher for $\mathrm{CD}$ than $\mathrm{AD}$ and depression was the most common comorbidity in the CD in this study. However, behavioral, verbal, developmental, and intellectual disorders, rather than mood disorders, might be common comorbidities in pediatric patients. ${ }^{19)}$ In addition, a meta-analysis of studies about co-occurrence of ADHD and unipolar depression in children and adolescents also emphasized that the interpretation of results can vary depending on the applied diagnostic standard; the number of subjects, the number of evaluators, and the length of the monitoring period. ${ }^{20)}$

An interesting finding from this study was the route of referral. For patients who visited a hospital during childhood, it was caregivers such as parents and teachers who recognized the symptoms, rather than the patients themselves. Patients who were first diagnosed in adulthood visited the hospital more frequently as self-referral due to media coverage. Ahmed et al. ${ }^{21)}$ claimed that media such as television shows and the internet portray ADHD medication negatively and therefore has a negative effect on parents' decision to seek medical treatment for children who suspect of exhibiting ADHD. However, in case of adult patients, the results from this study suggested that media should have played positive roles in the decision to visit the hospital. It means that public education and the development of an advertisement strategy are needed to convey accurate medical information and guidelines to the public and to connect them with treatment facilities when necessary.

The finding that the most common chief complaint at the first hospital visit was inattention for both group in this study, was consistent that attention deficit was the most important predictive factor for ADHD diagnosis in a large-scale adult cohort group. ${ }^{22)}$ Although hyperactivity is one of the main problems in childhood $\mathrm{ADHD},{ }^{2)}$ inattention was the commonest complaint in CD of this study. It might be related with tradition in Korea. ${ }^{23)}$ Because of the residue of son-preference in Korean culture, the parents are more tolerant of hyperactivity in boys. At the same time, parents are very ardent about the education of their children. This could be the reason why the parents are more intolerant of inattention, which affects the children's education, and more likely to take their children to hospital. Further studies are needed to verify this hypothesis.

There was no significant difference in subtypes between two groups, but inattentive type was most common for CD, while combined and inattentive type showed a similar distribution for AD. Fisher et al. ${ }^{24)}$ classified the subtype according to age in outpatients aged 9-80 who were diagnosed with ADHD between 1989 and 2009, and found that inattention was the most common symptom in all groups, including ages 9-14, 15-17, and over 18. In terms of ADHD subtype classification in adults, combined type was the most common subtype followed by the inattentive subtype. ${ }^{25)}$ Because the number of subjects in both groups was small in this study and as they are long-term treatment groups, the results of this study cannot reveal general age-related characteristics in the ADHD group.

It is controversial as to whether ADHD treatment can affect intelligence in adulthood, but a long-term study on natural progression in $\mathrm{ADHD}$ patients revealed that patients who were not treated during childhood showed a lower IQ in adulthood. ${ }^{26)}$ In an ADHD treatment compliance study, ${ }^{14)}$ children who terminated treatment early revealed that parents' education levels and the pediatric patients' IQ were higher compared to the group who maintained long-term treatment. That is, because pediatric patients with high intelligence discontinued treatment early, it is likely that the IQ of the CD in this study was lower than that of the AD. Pediatric patients with ADHD and high IQ can show similar accomplishment to children of average intelligence without ADHD, and ADHD-related problems in high IQ patients are not evident because of this. ${ }^{27)}$ Thus, ADHD treatment can be delayed or terminated early even if the treatment was initiated. In this study, high intelligence could be the reason that adults were first diag- 
nosed as ADHD without previous childhood diagnosis.

The fact that the AD showed significantly higher NS and HA subscale scores of the TCI than the CD in this study suggests an effect on ADHD characteristics that lasts until adulthood. Faraone et al. ${ }^{28)}$ reported significantly higher NS and HA in both patients with ADHD and late-onset ADHD (diagnosed over 7 years of age) compared to healthy individuals. Carlotta et al. ${ }^{29)}$ confirmed high scores on the NS in adults with ADHD, and patients with borderline personality disorder with a history of aggressiveness in adolescence. In that regard, the NS and HA subscales would be important indices for diagnosis of ADHD in adult as well as pediatric patients. In this study, scores on the SD and C subscales were also significantly lower for $\mathrm{AD}$ than $\mathrm{CD}$. Garcia et al. ${ }^{30)}$ revealed that $\mathrm{SD}$ and $\mathrm{C}$ factors could predict future dysfunction in autism spectrum disorder, ADHD, and learning disabilities. And the SD was significantly lower in the late-onset ADHD (diagnosed over 7 years of age) group than the subthreshold ADHD group. ${ }^{28)}$ The SD might be adjunctive for predicting symptoms and prognosis in ADHD.

This study has a few limitations that should be considered before generalizing its findings. First, when interpreting the study results, we should consider the disadvantage of the retrospective study; we relied on clinical evaluations rather than objective tools for characterizing chief complaints and comorbidities; the psychological tests like intelligence tests and TCI are different versions due to age difference of the subjects. Second, because this is a study of outpatients who visited one university hospital, the corresponding geographic, social, and demographic limitations must be considered. On the contrary, this limitation might also hold valid for certain people and valid for unique culture. Third, for patients who first visited a hospital in adulthood, childhood medical history and information for subtype diagnosis classification can be insufficient due to the limited recollection of past symptoms and history, and limited information gleaned from third-person accounts.

Despite such limitations, the strength of this study is that we reviewed the data of patients whose first diagnosis and longterm monitoring were conducted by the same expert trained as child and general psychiatrist. Regarding study design, this is the first study to compare patients who were diagnosed in childhood and patients diagnosed firstly in adulthood.

\section{CONCLUSION}

ADHD is one of disorders usually first diagnosed in infancy and childhood. The subjects in this study were adults who were diagnosed as ADHD in childhood and treated untill adulthood and adults who were not diagnosed as ADHD in child- hood. Though adults diagnosed first time in adulthood revealed inattentive and depressive symptoms as like as previous studies, higher IQ and predominance of male gender were the characteristics of the subjects. In practice, clinicians have to consider ADHD as one of psychiatric entity for adults without previous history of ADHD in childhood.

\section{Conflicts of Interest}

The authors have no financial conflicts of interest.

\section{REFERENCES}

1) American Psychiatric Association. Diagnostic and Statistical Manual of Mental Disorders. 4th ed. Washington, DC: American Psychiatric Association;1994.

2) American Psychiatric Association. Diagnostic and Statistical Manual of Mental Disorders. 5th ed. Washington, DC: American Psychiatric Association;2013.

3) Surman CB, Goodman DW. Is ADHD a valid diagnosis in older adults? Atten Defic Hyperact Disord 2017 Jan 25 [Epub ahead of print]. https://doi.org/10.1007/s12402-017-0217-x.

4) Taurines R, Schmitt J, Renner T, Conner AC, Warnke A, Romanos M. Developmental comorbidity in attention-deficit/hyperactivity disorder. Atten Defic Hyperact Disord 2010;2:267-289.

5) Balázs J, Keresztény A. Subthreshold attention deficit hyperactivity in children and adolescents: a systematic review. Eur Child Adolesc Psychiatry 2014;23:393-408.

6) Kim YS, Cheon KA, Kim BN, Chang SA, Yoo HJ, Kim JW, et al. The reliability and validity of Kiddie-Schedule for Affective Disorders and Schizophrenia-Present and Lifetime version- Korean version (K-SADS-PL-K). Yonsei Med J 2004;45:81-89.

7) Yoo HK, Lee J, Kang SH, Park EH, Jung J, Kim BN, et al. Standardization of the Comprehensive Attention Test for the Korean children and adolescents. J Korean Acad Child Adolesc Psychiatry 2009; 20:68-75.

8) Kwak K, Park H, Kim C. Korean Wechsler Intelligence Scale for Children-III (K-WISC-III). Seoul: Special Education Publishing Co.; 2001.

9) Yum TH, Park YS, Oh KJ, Kim JG, Lee YH. The Manual of KoreanWechsler Adult Intelligence Scale. Seoul: Korea Guidance;1992.

10) Min BB, Oh HS, Lee JY. Manual of Temperament and Character Inventory. Seoul: Maumsarang;2007.

11) Kessler RC, Adler L, Ames M, Demler O, Faraone S, Hiripi E, et al. The World Health Organization Adult ADHD Self-Report Scale (ASRS): a short screening scale for use in the general population. Psychol Med 2005;35:245-256.

12) Bahn GH, Ryu JE, Lee YJ, Han J, Lee A, Hong M. When is the endpoint of the adolescence? Psychoanalysis 2015;26:3-17.

13) Hong M, Kwack YS, Joung YS, Lee SI, Kim B, Sohn SH, et al. Nationwide rate of attention-deficit hyperactivity disorder diagnosis and pharmacotherapy in Korea in 2008-2011. Asia Pac Psychiatry 2014;6:379-385.

14) Hong M, Lee WH, Moon DS, Lee SM, Chung US, Bahn GH. A 36 month naturalistic retrospective study of clinic-treated youth with attention-deficit/hyperactivity disorder. J Child Adolesc Psychopharmacol 2014;24:341-346.

15) Goto T, Hirata Y, Takita Y, Trzepacz PT, Allen AJ, Song DH, et al. Efficacy and safety of atomoxetine hydrochloride in Asian adults with ADHD. J Atten Disord 2017;21:100-109.

16) Seo JY, Lee CS, Park CS, Kim BJ, Cha BS, Lee SJ, et al. Mediating effect of depressive symptoms on the relationship between adult attention deficit hyperactivity disorder and quality of life. Psychiatry Investig 2014;11:131-136.

17) Bhang SY, Kwack YS, Joung YS, Lee SI, Kim B, Sohn SH, et al. Fac- 
tors that affect the adherence to ADHD medications during a treatment continuation period in children and adolescents: a nationwide retrospective cohort study using Korean Health Insurance data from 2007 to 2011. Psychiatry Investig 2017;14:158-165.

18) Chen MH, Su TP, Chen YS, Hsu JW, Huang KL, Chang WH, et al. Higher risk of developing major depression and bipolar disorder in later life among adolescents with asthma: a nationwide prospective study. J Psychiatr Res 2014;49:25-30.

19) Jensen CM, Steinhausen HC. Comorbid mental disorders in children and adolescents with attention-deficit/hyperactivity disorder in a large nationwide study. Atten Defic Hyperact Disord 2015;7:27-38.

20) Meinzer MC, Pettit JW, Viswesvaran C. The co-occurrence of attention-deficit/hyperactivity disorder and unipolar depression in children and adolescents: a meta-analytic review. Clin Psychol Rev 2014;34:595-607.

21) Ahmed R, McCaffery KJ, Aslani P. Factors influencing parental decision making about stimulant treatment for attention-deficit/hyperactivity disorder. J Child Adolesc Psychopharmacol 2013;23: 163-178.

22) Matte B, Anselmi L, Salum GA, Kieling C, Gonçalves H, Menezes A, et al. ADHD in DSM-5: a field trial in a large, representative sample of 18- to 19-year-old adults. Psychol Med 2015;45:361-373.

23) Kim YJ, Oh SY, Lee J, Moon SJ, Lee WH, Bahn GH. Factors affecting adherence to pharmacotherapy in children with attention-deficit hyperactivity disorder:a retrospective study. J Korean Acad Child Adolesc Psychiatry 2010;21:174-181.

24) Fisher BC, Garges DM, Yoon SY, Maguire K, Zipay D, Gambino M, et al. Sex differences and the interaction of age and sleep issues in neuropsychological testing performance across the lifespan in an ADD/ADHD sample from the years 1989 to 2009. Psychol Rep 2014; 114:404-438.

25) Sobanski E, Brüggemann D, Alm B, Kern S, Philipsen A, Schmalzried H, et al. Subtype differences in adults with attention-deficit/ hyperactivity disorder (ADHD) with regard to ADHD-symptoms, psychiatric comorbidity and psychosocial adjustment. Eur Psychiatry 2008;23:142-149.

26) Rasmussen P, Gillberg C. Natural outcome of ADHD with developmental coordination disorder at age 22 years: a controlled, longitudinal, community-based study. J Am Acad Child Adolesc Psychiatry 2000;39:1424-1431.

27) Antshel KM, Faraone SV, Stallone K, Nave A, Kaufmann FA, Doyle A, et al. Is attention deficit hyperactivity disorder a valid diagnosis in the presence of high IQ? Results from the MGH Longitudinal Family Studies of ADHD. J Child Psychol Psychiatry 2007;48:687694.

28) Faraone SV, Kunwar A, Adamson J, Biederman J. Personality traits among ADHD adults: implications of late-onset and subthreshold diagnoses. Psychol Med 2009;39:685-693.

29) Carlotta D, Borroni S, Maffei C, Fossati A. On the relationship between retrospective childhood ADHD symptoms and adult BPD features: the mediating role of action-oriented personality traits. Compr Psychiatry 2013;54:943-952.

30) Garcia D, Anckarsäter H, Lundström S. Self-directedness and cooperativeness, psychosocial dysfunction and suffering in ESSENCE. ScientificWorldJournal 2013;2013:416981. 